\title{
Reorganization of the Chick Basilar Papilla After Acoustic Trauma
}

\author{
YEHOASH RAPHAEL \\ Kresge Hearing Research Institute, The University of Michigan, Ann Arbor, \\ Michigan 48109-0506
}

\begin{abstract}
The auditory epithelium in birds and mammals consists of a postmitotic population of hair cells and supporting cells. Unlike mammals, birds can regenerate their auditory epithelia after trauma. Recent evidence indicates that supporting cells undergo mitosis after acoustic trauma, suggesting that supporting cells may transdifferentiate into hair cells. The goals of this study were to 1) characterize the responses of hair cells and supporting cells to acoustic trauma, and 2) determine whether hair cell loss is a prerequisite for generation of new hair cells. Chicks were exposed to an octave-band noise and their inner ears assayed with fluorescence or scanning electron microscopy. In one area of the basilar papilla, defined as the center of the lesion, extensive hair cell degeneration occurred. Expanded supporting cells obliterated degenerating hair cells and invaded spaces normally occupied by hair cells. Aggregates of DNA were found within the basilar papilla, suggesting that hair cell death and disintegration may occur within the epithelium. The epithelial sheet appeared structurally confluent at all times examined. Supporting cells exhibited altered apical contour in distal regions of the basilar papilla, where hair cell damage was mild or inconspicuous. Four days after noise exposure, newly generated hair cells were found in the center of the lesion and in the distal areas, where no hair cell loss could be detected. The results suggest that supporting cells may play an important role in maintenance and repair of the traumatized basilar papilla and raise the possibility that production of new hair cells is not dependent on hair cell loss in the immediate vicinity. 1993 Wiley-Liss, Inc.
\end{abstract}

Key words: noise, repair, regeneration, mitosis

In chick, hair cells that are lost after acoustic or chemical trauma are replaced by new, normal appearing hair cells (Cotanche, '87; Corwin and Cotanche, '88; Ryals and Rubel, '88). The mechanisms that initiate and control this regenerative process are largely unknown.

Mechanisms that control regeneration vary between different sensory epithelial tissues. In the experimentally damaged teleost fish eye, rod precursors can give rise to more than one retinal cell type, suggesting that cues from the surroundings influence the regenerative process (Raymond, '91). Experiments with cultured aggregates of chick retinal pigment epithelium have shown that transdifferentiation of these cells is modulated by cell shape, the composition of the extracellular matrix, and the presence of bFGF (reviewed in Reh et al., '91). In the olfactory neuroepithelium, degenerated neurons can be replaced by new neurons that arise via mitotic divisions of basal cells (Graziadei and Monti Graziadei, '85). The possibility that extracellular matrix proteins and growth factors regulate growth and differentiation in olfactory neuroepithelium has also been investigated (Calof et al., '91), but the mechanism of regulation has yet to be determined. In the postembryonic oscar (Astronotus ocellatus), a teleost fish, "embryonic like" neuroepithelial cells serve as stem cells, which can give rise to new hair cells and supporting cells (Presson and Popper, '90). Nevertheless, it is not known how proliferation and regeneration of new hair cells and supporting cells are regulated. Thus, data at the cellular, molecular, and genetic levels indicate that control mechanisms that regulate epithelial regeneration are species and tissue specific.

${ }^{3} \mathrm{H}$ thymidine studies in traumatized chick inner ears showed that labeled hair cells and supporting cells were present in the basilar papilla (BP) after several days of recovery from acoustic overstimulation (Corwin and $\mathrm{Co}^{-}$ tanche, '88; Girod et al., '89). Thus, cell divisions appear to play a role in repopulating the BP after trauma. Recent data showing that supporting cells divide within 2 days after trauma (Raphael, '92) have implicated supporting cells as a source of newly generated hair cells. The signal(s) regulat-

Accepted December 23, 1992.

Preliminary results were presented in part at the First Meeting on Molecular Biology of Hearing and Deafness, San Diego, May 1992, and the 29th Workshop on Inner Ear Biology, Engelberg, September 1992. 
ing supporting cell proliferation and differentiation in the noise exposed BP are not known.

To explain the regulatory mechanism that controls generation and differentiation of new cells in the basilar papilla, Corwin et al. ('91) suggested that presence of intercellular contacts with hair cells inhibits supporting cells from proliferating, According to this hypothesis, loss of two or more hair cells would deprive supporting cells of heterophilic contacts, leading to removal of lateral inhibition and a subsequent regenerative response.

The goals of this study were 1) to characterize the process of hair cell degeneration and the relationships between hair cell degeneration and the response of supporting cells, and 2) to test the hypothesis that changes in cell-cell contacts that result from the loss of hair cells and their replacement by expanded supporting cells are necessary to induce proliferation of supporting cells. To that end, the responses of supporting cells and hair cells to acoustic trauma were documented by fluorescence or scanning electron microscopy on wholemounts of the BP.

\section{MATERIALS AND METHODS Animals and noise exposure}

One week old white Leghorn chick hatchlings were placed for 4 hours in a mesh-wire cage beneath a horn speaker that delivered octave-band noise with a center frequency of 1.5 $\mathrm{kHz}$ and intensity of $120 \mathrm{~dB}$ SPL. The signal was generated by a random noise generator (General Radio Company, model 1381), filtered, and amplified (MC 2105, McIntosh Power Amplifier). Intensity was calibrated with a Precision Sound Level Meter (type 2203, Bruel \& Kjaer). Chicks were killed at $0(n=10), 6(n=5), 24(n=4), 48(n=8)$, or 96 $(n=6)$ hours after noise exposure. Seven chicks were used as untreated controls.

\section{Histo- and immunocytochemistry}

Chicks were anesthetized and perfused intracardially with $3 \%$ paraformaldehyde in $0.15 \mathrm{M}$ phosphate buffer at $\mathrm{pH}$ 7.35. Temporal bones were rapidly removed and $\mathrm{BP}$ harvested. Tissues were prepared as wholemounts, permeabilized with $0.1 \%$ Triton X-100 in phosphate buffered saline (PBS) for 5 minutes and stained with one or more of the following probes: cingulin-specific antibodies were used to label tight-junctions, phalloidin to stain $\mathrm{F}$-actin, and bisbenzimide trihydrochloride (Hoechst) to label DNA. Nonspecific immunoreactivity was blocked in normal goat serum (5\% in PBS) for 30 minutes. Samples were then incubated in rabbit anti-cingulin antibody (1:600 dilution; Citi et al., '88, '89) for 60 minutes, rinsed thoroughly, and immersed in a PBS solution containing Hoechst $(2 \mathrm{mg} / \mathrm{ml}$, Sigma), rhodamine phalloidin (1:100; Molecular Probes, $\mathrm{OR}$ ), and FITC-conjugated goat anti rabbit secondary antibody (1:100; Cappel, Durham, NC) for 30 minutes. After rinsing, papillae were mounted in $60 \%$ glycerol in sodium carbonate buffer ( $\mathrm{pH} 8.5$ ) with $p$-phenylenediamine as an anti-bleach agent. Controls for specificity of labeling included omission of primary antibodies and labeling of several nonauditory tissues.

Preparations were photographed with a Leitz Orthoplan microscope equipped for epifluorescence with $\times 50$ and $\times \mathbf{1 0 0}$ oil objectives, or with a Bio-Rad laser confocal microscope with an $\times 100$ oil objective. Photography was performed with Kodak T-max 400 film exposed at 1,600 ASA.

\section{Scanning electron microscopy (SEM)}

Animals were fixed as described above for histochemistry and then postfixed in $1 \%$ aqueous osmium tetroxide for 30 minutes. Wholemounts were processed using routine methods (Raphael, '92). Tissues were analyzed and photographed with an AMRAY 1000B scanning electron microscope operated at $10 \mathrm{kV}$.

\section{RESULTS}

The use of wholemounts for histochemical analysis enabled visualization of the BP in its entirety (Fig. 1A). Using wholemounts with double- and triple-labeling proved to be an excellent method for assessing the tissue organization, distribution of junctional complexes, and location and integrity of nuclei. In control tissues, F-actin (microfilaments) was present in the stereocilia and the cuticular plate (terminal web) of every hair cell (Fig. 1B), as previously described (Raphael, '91). Hair cells were normally organized in rows with similar spaces between each other. Co-localization of actin (Fig. 1B) and Hoechst (Fig. 1C) in control tissues made it possible to locate the nucleus in relation to the actin cytoskeleton of each cell. Normal hair cells exhibited round nuclei organized in rows, visible at a focal plane immediately beneath the luminal surface. Supporting cell nuclei also labeled by Hoechst stain were located at a deeper focal plane closer to the basement membrane (not shown). Thus, nuclei of cells in the BP had a laminar distribution, with hair cell nuclei immediately beneath the reticular lamina (RL) and supporting cell nuclei beneath them, closer to the basal lamina, as previously reported (Raphael, '92). Cingulin (Fig. 1D), a protein specific to tight-junctions (Citi et al., ' 88 , ' 89 ), appeared as a narrow line at the focal plane of the RL. Thus, cingulinspecific label was found in homophilic (supporting cellsupporting cell) and heterophilic (support cell-hair cell) junctions at the RL. Because the apical surface area of supporting cells was very slender, cingulin label often appeared as a single line. Nevertheless, it should be noted that cingulin was present all around the apical contour of supporting cells. Therefore, the pattern of cingulin distribution was a good marker to delineate the polygonal apical contours of hair cells and the narrow apical contour of supporting cells (Figs. 1D, 4A). In the polygon that surrounds each hair cell, contact points between adjacent supporting cells may be at the sharp corners, as depicted in Figure $4 \mathrm{~A}^{\prime}$, or, alternatively, along the sides of the polygon.

Actin and cingulin were co-localized at the apical intercellular contacts of cells in the BP (Fig. $1 \mathrm{E}$ and D, respectively). The pattern of cingulin and actin labels revealed similar cellular organization of the BP in the proximal and distal areas. The organization of the normal sensory mosaic of the avian BP, shown with two fluorescent markers (Figs. $1 D, E)$, is consistent with previous reports based on scanning electron microscopy (Hirokawa, '78; Tanaka and Smith, '78; Chandler, '84).

Structural alterations that accompany hair cell degeneration were consistently observed in noise exposed chicks in a region located $1.2 \mathrm{~mm}$ from the proximal end of the $\mathrm{BP}$. This region of damage is referred to as the "center of the lesion" throughout this work (see Fig. 1A for location of this region). Supporting cells in the center of the lesion exhibited an expanded apical area, and occupied a significant portion of the total surface of the RL, as previously described (Cotanche, '87; Cotanche and Dopyera, '90; Marsh 

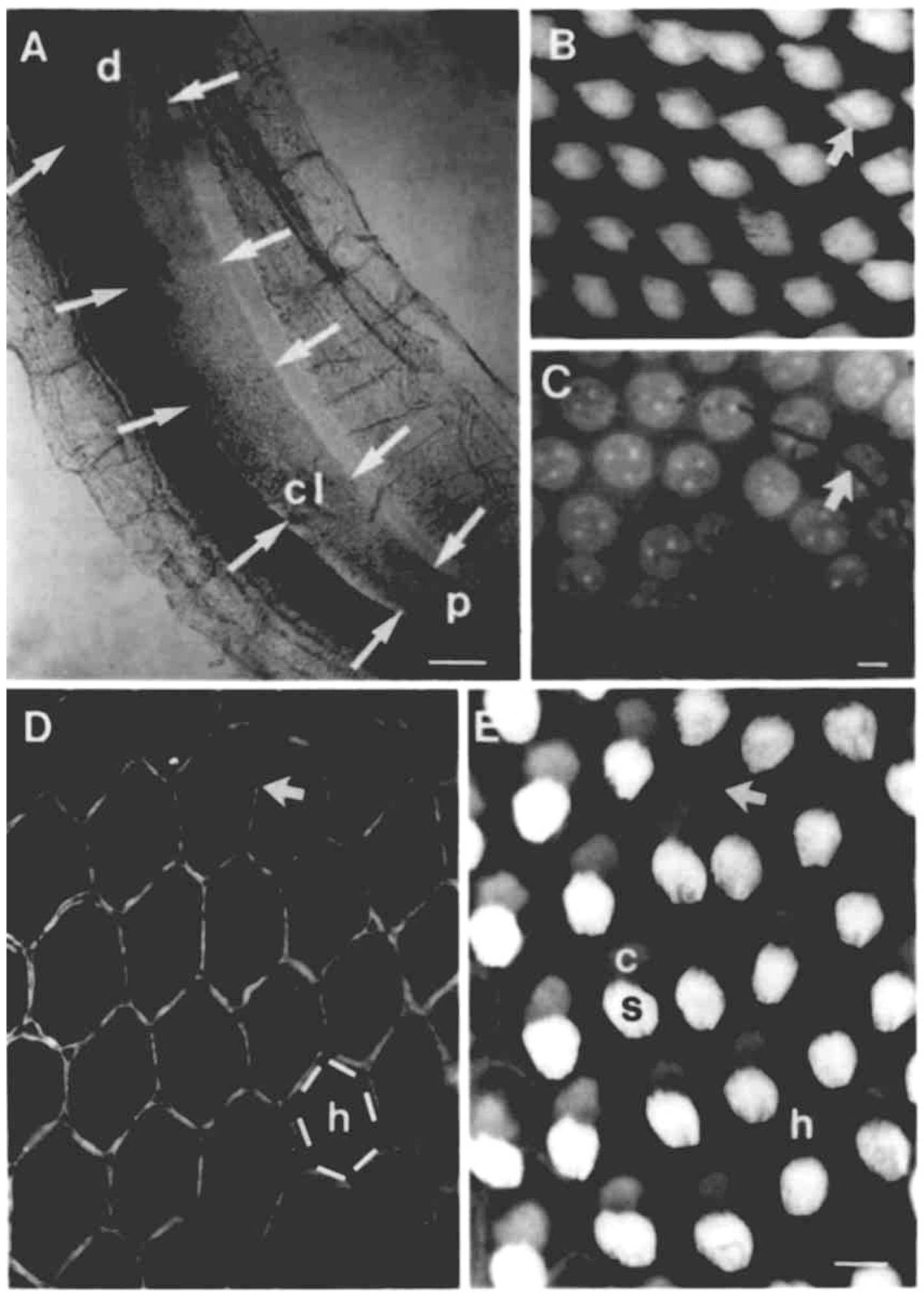

Fig. 1. Wholemounts of control (unexposed) chick basilar papilla viewed in phase-contrast microscopy (A) or fluorescence microscopy with probes for actin (B and E), DNA (C), or cingulin (D). A: The basilar papilla and adjacent tissues are oriented with the proximal part (p) at the bottom of the micrograph and the distal (d) regions at the top left. The neural and abneural margins of the basilar papilla are marked by arrows. The abneural side is on the right. The center of the lesion (cl) induced in this work (described in the following figures) is close to the proximal region of the basilar papilla. The extreme proximal tip is not in the photographed field. B,C: Actin label reveals hair cells in organized rows (B) and Hoechst in same field shows hair cell nuclei (C). Arrows in $\mathrm{B}$ and $\mathrm{C}$ point to the same cell though the focal plane is slightly different. D,E: Co-localization of cingulin and actin. Cingulin expres-

sion (D) is restricted to tight junctions, connecting the apical surfaces of all cells in the basilar papilla. Slender double lines (arrow) on sides of hair cells delineate supporting cells that surround every hair cell, forming a polygonal shape with 5 or 6 sides $(\mathrm{h}$, apical surface of a hair cell surrounded by 6 supporting cells). Actin label $(E)$ is in the normally packed bundles of stereocilia (s) and in the cuticular plate (c) of hair cells (h marks the apical surface of the same hair cell in $\mathrm{E}$ and $\mathrm{D}$ ). Actin label is also in a belt of adherens junctions (arrow). Arrows in D and E point to identical freld, showing tight junctions around a supporting cell in $D$ and actin in adherens junctions of the same junctional complex in E. Abneural side of basilar papilla faces top in B-E. Bars: $250 \mu \mathrm{m}$ in A; 5 $\mu \mathrm{m}$ in $\mathrm{C}$ and $\mathrm{E}$ 

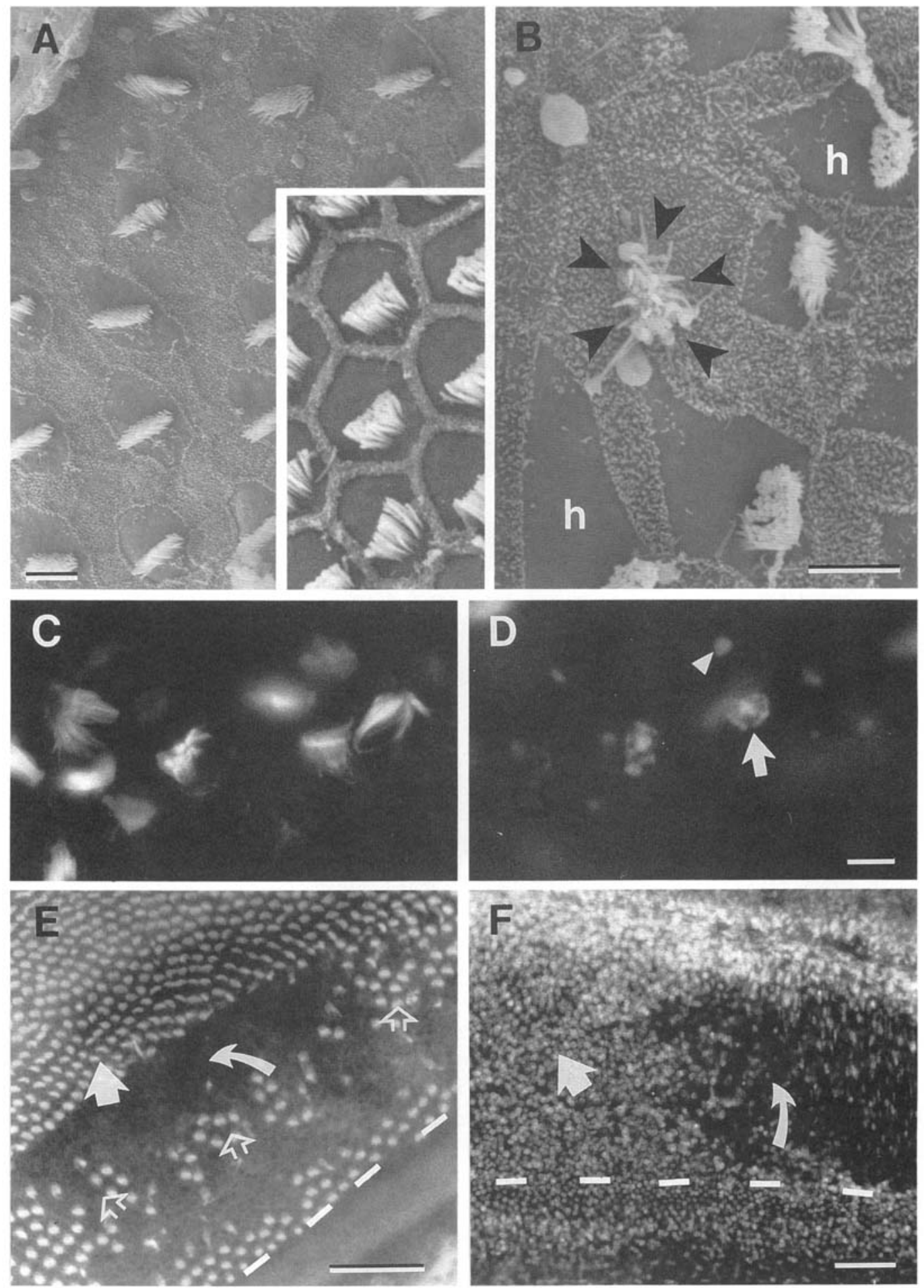

Fig 2. SEM (A, B) and fluorescence microscopy (C-F) photomicrographs of lesioned area in basilar papilla of chicks exposed to noise for 4 hours. Inset in A shows the normal apical organization of the basilar papilla, in a control specimen. A: Immediately after exposure the apical surfaces of hair cells are smaller than normal, whereas supporting cells are expanded and occupy more space than they normally do. Most hair cells have a circular-shaped apical surface. The stereocilia appear normal, and the orderly rows of hair cclls are maintained with no apparent hair cell loss. A-inset: In control tissue, the apical membranes of hair cells occupy most of the surface area of the basilar papilla, whereas the apical extensions of supporting cells form straight and nerrow lines that appear as a polygon around hair cells. Note that the distance between centers of stereocilia bundles on neighboring hair cells is similar in exposed and control tissues. B: Six hours after noise exposure several hair cells (h) still remain in the center of the lesion. The apical surfaces of some hair cells (areas devoid of microvilli) are severely constricted. One hair cell is completely obliterated by invading supporting cells (arrowheads), so that only the stereacilia protrude from the reticular lamina. C,D: Co-localization of actin (focused at stereocilia, in C) and Hoechst (focal plane of hair cell nuclei, in D), showing hair cell loss and damaged hair cells $(C)$ and missing nuclei and degenerating nuclei (arrow) (D) 6 hours after noise exposure. DNA aggregates (arrowhead) are scattered within the epithelium. E: Low magnification of the lesion 6 hours after noise exposure reveals extensive hair cell loss in the center of the lesion (curved arrow points to the region with complete hair cell loss). In some areas in the lesion several hair cells were present (open arrows) and in more remote regions no hair cell loss is seen (arrow). White bars mark the abneural border of the basilar papilla. F: Six hours after exposure, many hair cell nuclei are missing in the lesioned area (curved arrow), whereas a normal distribution of nuclei is observed in the more distal region (arrow). The abncural side of basilar papilla faces the bottom of the micrographs. White bars mark the abneural border of the basilar papilla. Bars: $5 \mu \mathrm{m}$ in A (for A and inset), B, and D; $50 \mu \mathrm{m}$ in $\mathrm{E}$ and $\mathrm{F}$. 

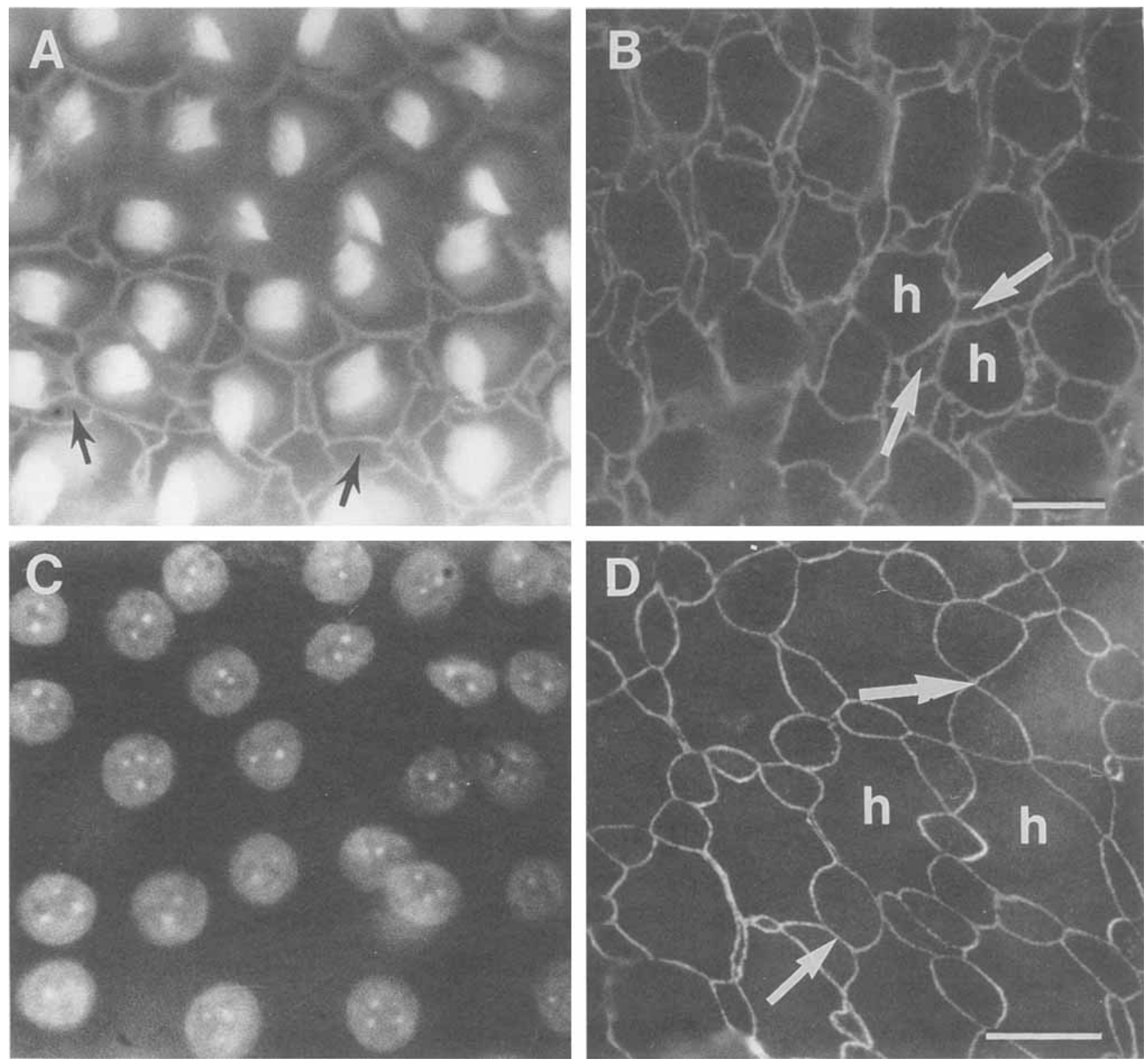

Fig. 3. Distal basilar papilla immediately after noise exposure (A-C) or 6 hours after exposure (D), labeled for actin (A), cingulin (B, D), or DNA (C). The abneural side of the basilar papilla faces left side in all micrographs. A: Actin is distributed normally in stereocilia and the cuticular plate of hair cells. Actin in adherens junctions delineates the borders of cells in the reticular lamina and reveals that the surface area of hair cells is slightly reduced, whereas supporting cells in the lower half of micrograph (arrows) are expanded (compare to control in Fig. 1E). There is a sharp transition between the region with expanded supporting cells (in the lower part of the micrograph) and the undamaged region (in the upper part of the micrograph). Note that all hair cells are present and bundles of stereocilia appear intact. B: Cingulin distribution shows tight junctions at the reticular lamina. Hair cells $(h)$

et al., '90; Raphael, '92). The apical surface area of hair cells was reduced, relative to normal, immediately after the noise exposure (Fig. 2A, compare to control in inset), as previously described by Marsh et al. ('90). In addition to being constricted, the apical contour of hair cells in the center of the lesion appeared oval or circular, instead of the

are smaller than normal (compare to control in Fig. 1D). Many supporting cells that border the sides of hair cells are expanded and appear as a "figure eight" (arrows) or as 2 rings sharing a common middle line. Note that no hair cell loss is seen. C: The distribution of Hoechst stain reveals no loss of hair cell nuclei. The orderly rows are generally preserved, although 2 muclei on the right side of micrograph are slightly out of place. D: Cingulin expression 6 hours after exposure shows more pronounced supporting cell expansion. Hair cell contours are irregular due to supporting cell bulging. Areas of constriction between the 2 halves of each figure eight appear as lines of cingulin (small arrow) or as crossover points (large arrow). Bars: $10 \mu \mathrm{m}$ in $B$ (for $\mathrm{A}$ and $\mathrm{B}$ ); $10 \mu \mathrm{m}$ in $\mathrm{D}$ (for $\mathrm{C}$ and $\mathrm{D}$ ).

normally occurring polygonal contour. Stereocilia on hair cells with constricted surface often appeared normal (Fig. 2A).

Six hours after noise exposure, hair cells in the center of the lesion had very constricted apical surfaces and supporting cells were extremely expanded (Fig, 2B). Hair cells that 


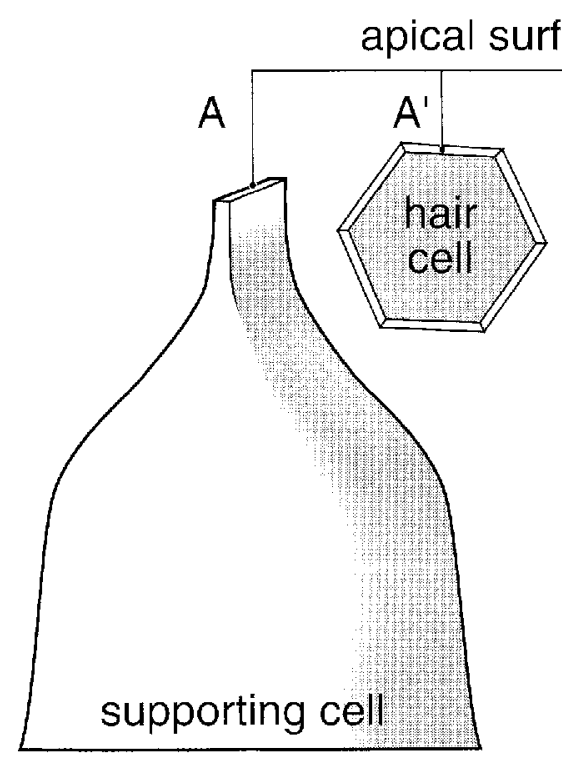

Fig. 4. A schematic representation of the cellular elements that construct the reticular lamina in control basilar papilla $\left(\mathbf{A}, \mathbf{A}^{\prime}\right)$ and in noise exposed basilar papilla at the distal region ( $\mathrm{B}, \mathrm{B})$. A: The basal pole of supporting cells is in contact with the basal lamina and the apical pole extends to the RL. The apical membrane of each normal supporting cell appears like a slender line. A': Top view, showing the apical surfaces of supporting cells that border a hair cell, together forming a polygon. Six (or, occasionally, five) supporting cells construct the polygon, but it is not clear whether contact points between these

were nearly completely engulfed by expanded supporting cells could often be identified by the remaining stereocilia that protruded from the RL (Fig. 2B). In many cases, all 5-6 supporting cells surrounding a hair cell were expanded and appeared to invade the spaces normally occupied by hair cells.

A substantial reduction in the number of nuclei in the lesion was revealed by the distribution of Hoechst stain in the lesion 6 hours after the noise exposure (Fig. 2D; compared to Fig. $1 \mathrm{C}$ ). This reduction was noted at the focal plane of hair cell nuclei. In this area, actin distribution revealed damage to hair cells, whereas Hoechst stain showed degenerating nuclei as well as scattered DNA aggregates (Fig. 2C,D), suggesting that cells were disintegrating in situ. At this stage, phalloidin-labeled tissues revealed a lesion with extensive hair cell loss (Fig. 2E). Inspection of Hoechst-labeled BP at this stage revealed that in the area of lesion, very few nuclei remained at the focal plane just beneath the RL (Fig. 2F), confirming that hair cells were missing.

The region referred to as "distal BP" throughout this work was located distally to the center of the lesion, approximately $2 \mathrm{~mm}$ from the proximal end of the BP. The distal BP exhibited moderate structural alterations immediately after noise exposure. The distribution of actin in the stereocilia and cuticular plate of hair cells was unchanged in the distal BP after noise exposure (compare Fig. 3A to Fig. 1E). However, the relative size of hair cells and supporting cells was altered, as was the shape of supporting cells (Fig. 3A,B). Cingulin-specific label at the apical contour of supporting cells showed that these supporting cells were expanded, whereas apical surfaces of the hair cells were smaller than normal and irregularly shaped (compare
Fig. 3B to Fig. 1D). The most distal part of the BP did not display morphological changes, and the border between affected and unaffected regions in the distal BP was clearly observed (Fig. 3A). The pattern of cingulin distribution in the distal BP after noise exposure showed hair cells organized in rows as in the normal BP, and did not provide evidence for hair cell loss. Based on the distribution of cingulin, supporting cells in the distal BP appeared wider than normal at their apical surfaces and in many cases the contour of their apical membranes resembled "figure eights" (Figs. 3B, 4B). The distribution of Hoechst in the distal BP revealed that all hair cell nuclei were present, although some nuclei were slightly dislocated relative to normal (Fig. 3C). Degenerating nuclei were not observed in the distal BP. The distribution of cingulin in the distal BP 6 hours after noise exposure (Fig. 3D) was similar to that observed immediately after noise exposure (see Fig. 4 for schematic). In some areas, the apical contour of each supporting cell was composed of two rounded portions, forming a figure eight. Hair cells were slightly constricted and their side borders were irregularly shaped. Nevertheless, hair cell loss was not observed in the distal BP.

In the center of the lesion, expanded supporting cells with a thick cover of microvilli occupied a large region with extensive hair cell loss 24 hours after noise exposure (Fig. 5A). A few hair cells with stereocilia bundles and a smooth apical membrane survived the trauma and remained in the region, among expanded supporting cells. In some cases, the remains of hair cells were visible at the surface of the epithelium. In contrast, no hair cell loss occurred in the distal BP (Fig. 5B). Nevertheless, supporting cells in the distal BP were slightly expanded and their apical surface resembled a figure eight (compare Fig. 5B to Fig. 3D). 

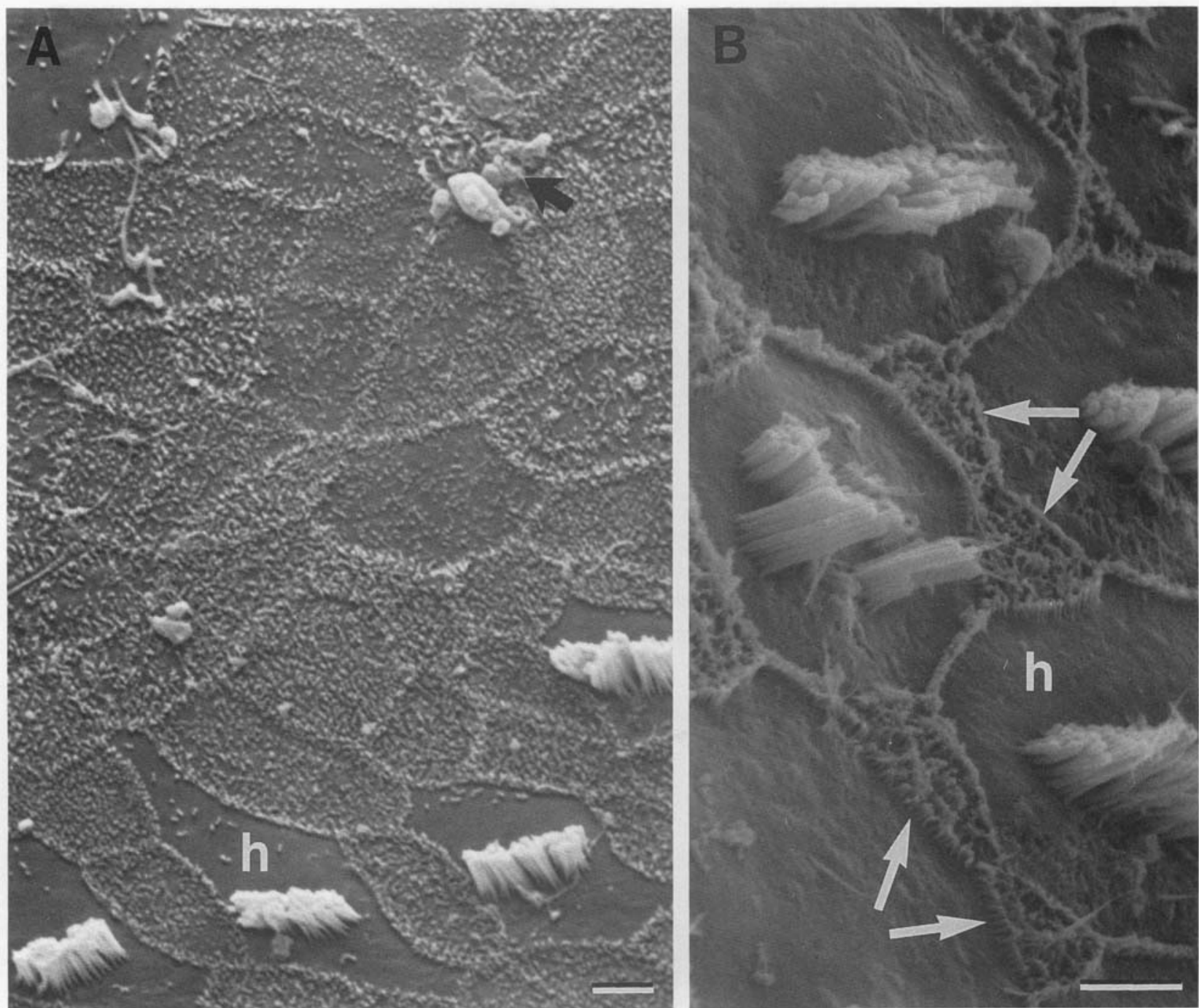

Fig. 5. SEM of the center of lesion (A) and the distal portion of basilar papilla (B) 24 hours after noise exposure. A: Several surviving hair cells (h) remain in the lesion, whereas numerous expanded supporting cells cover an extensive region with hair cell loss. Stereocilia of a hair cell in final stage of degeneration protrude from the surface

During all stages of hair cell degeneration, the confluence of the $\mathrm{RL}$ was uninterrupted, and protrusion or ejection of hair cell bodies from the RL was not observed.

Forty-eight hours after noise exposure, the center of the lesion contained a few hair cells with features of noisedamaged cells. These hair cells had a large apical surface and a well developed, although irregularly arranged, bundle of stereocilia, suggesting that they survived the trauma (Fig. 6A). These surviving (preexisting) hair cells were surrounded by supporting cells with expanded apical surfaces (Fig, 6A). At this time, mitotic chromosomes were found in the lesion at a focal plane immediately beneath the RL (Fig. 6B), as previously described (Raphael, '92). Cells that contained mitotic chromosomes (Fig. 6C) exhibited spherical cell bodies (Fig. 6D). However, the distribution of actin in the junctional complexes at the level of the RL directly above the dividing chromosomes did not disclose a round or spherical cell contour (Fig. 6E), suggesting that the apical surface of dividing cells is not necessarily round. (arrow). B: No hair cell loss is seen in the distal basilar papilla, and stercocilia on most cells are normal, though some bundles are slightly damaged. Expanded supporting cells shaped as a figure eight (arrows) are positioned between hair cells. Bars: $2 \mu \mathrm{m}$.

Ninety-six hours after noise exposure, hair cells with a small, embryonic-like apical surface were observed in the center of the lesion (Figs. 7A, 8A) and in the distal BP (Figs. $7 \mathrm{~B}, 8 \mathrm{~B})$. Small hair cells were considered to be new based on their size and shape. Thus, cells with (apical) surface area not exceeding half that of normal (mature) hair cells, and immature organization of stereocilia, were considered to be newly generated hair cells.

In the center of the lesion, expanded supporting cells formed the immediate borders of new hair cells (Figs. 7A, $8 \mathrm{~A}$ ). In the distal BP (Figs. 7B, 8B), hair cell loss was not evident, and new hair cells were situated among preexisting hair cells.

Most, but not all of the newly added hair cells were found in pairs in the lesioned as well as the distal BP. As soon as they could be recognized as hair cells, regenerated hair cells were appropriately oriented, with the kinocilia and the tallest stereocilia at the abneural pole of the cell. 

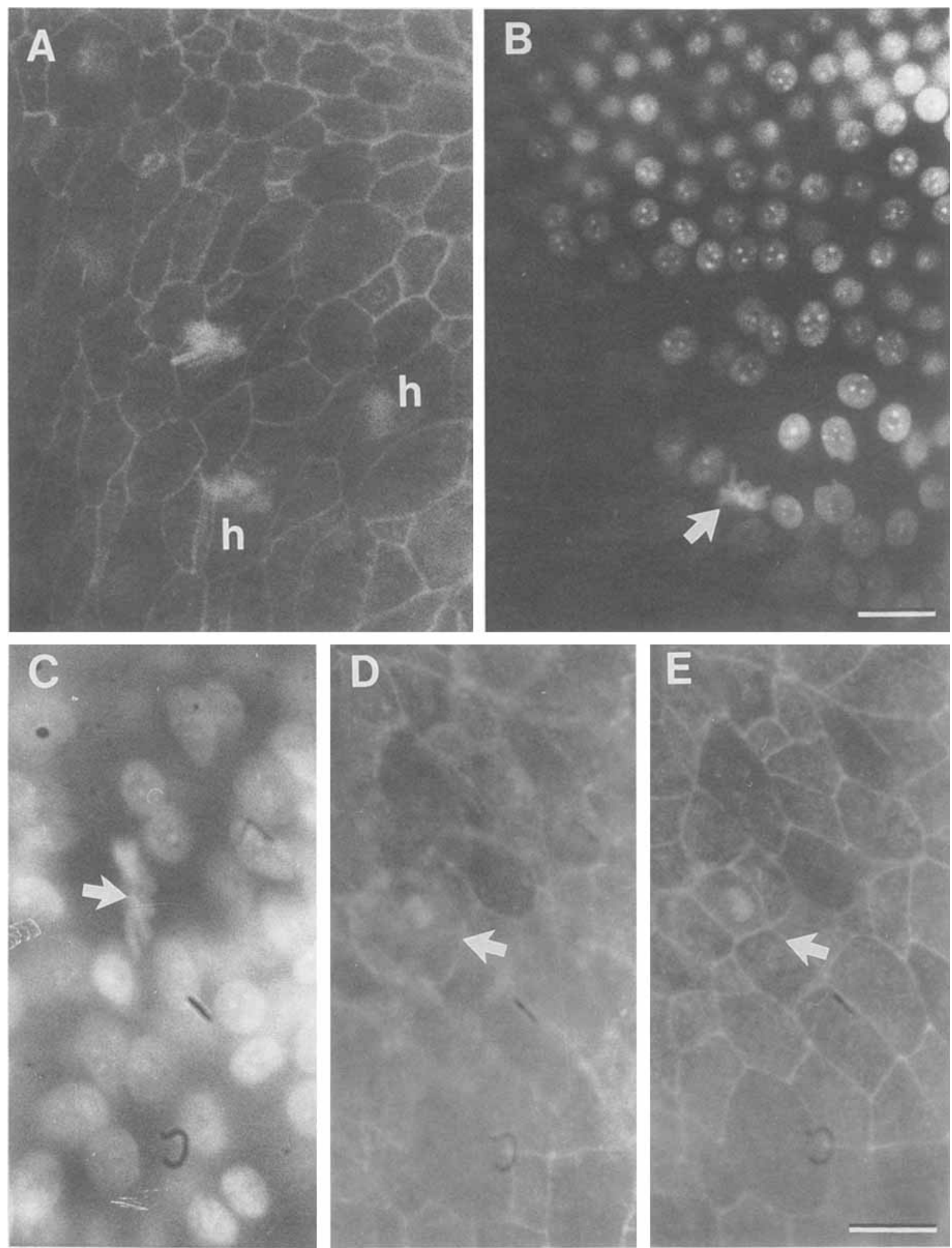

Fig. 6. Fluorescence micrographs of center of lesion in basilar papilla 48 hours after noise exposure. A: Actin label shows extensive hair cell loss, although a few surviving hair cells (h) remain. B: DNA label shows mitotic chromosomes (arrow) in lesion, at focal plane immediately beneath reticular lamina. C,D: Mitotic chromosomes (arrow in $\mathrm{C}$ ) are enclosed by a belt of actin label (in D), showing the same region as $\mathrm{C}$ ), showing rounded shape of dividing cell. E: The same region shown in $\mathrm{D}$ and $\mathrm{F}$, but at focal plane of the reticular lamina. Several lines of actin traverse the region directly above the dividing chromosomes. In the area where division occurs, no remaining hair cells are seen. Arrows point to the same area in D and E. Bars: $10 \mu \mathrm{m}$ in $\mathrm{B} ; 10 \mu \mathrm{m}$ in $\mathrm{E}$ (for $\mathrm{A}, \mathrm{C}, \mathrm{D}, \mathrm{E}$ ). 


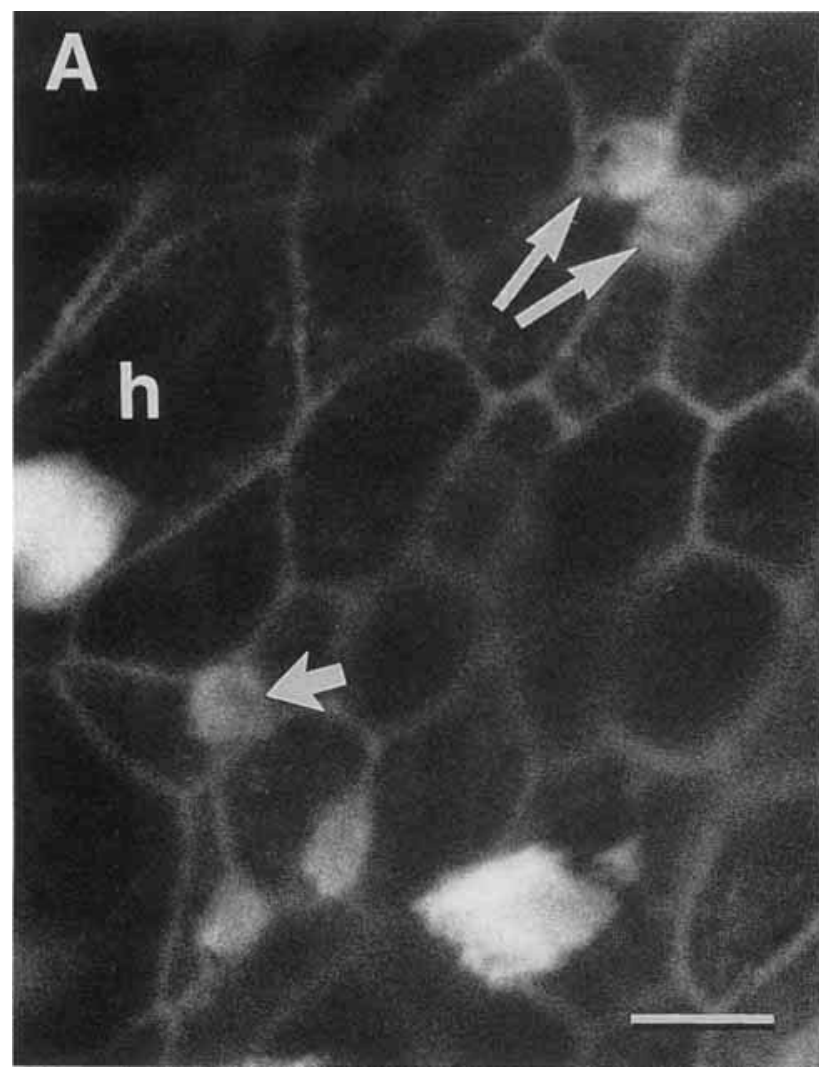

Fig. 7. Actin distribution in the center of lesion (A) and in the distal basilar papilla (B) 96 hours after noise exposure. A: Fluorescence micrograph of the center of lesion where small hair cells with immature appearance are found among expanded supporting cells and surviving hair cells (h). Some immature hair cells appear in pairs (long arrows)

\section{DISCUSSION \\ Supporting cell expansion and hair cell degeneration}

Expansion of supporting cells in the BP after noise exposure was first described by Cotanche et al. ('87) and later reported in additional works (Cotanche, '87; Marsh et al., '90; Raphael and Altschuler, '92). The mechanism of supporting cell expansion is still unknown. In this work, supporting cells expanded not only in areas of hair cell loss but also in remote areas without hair cell loss, suggesting that hair cell loss is not a prerequisite for supporting cell response to acoustic trauma.

The data presented here provide strong evidence that hair cells may degenerate without extrusion into the luminal space. This evidence is based on two observations. First, partly obliterated hair cells were observed protruding from the center of a cluster of supporting cells, suggesting that supporting cell expansion may trap hair cell bodies within the epithelium. Second, small aggregates of DNA were found in the region where hair cells degenerate after noise exposure, strongly suggesting that cell death occurs within the epithelium. Since supporting cells do not appear to degenerate after noise exposure, aggregates of DNA are most probably the remains of hair cell nuclei that disintegrate in situ.

Studies on hair cell degeneration in the organ of Corti have also indicated that expanding supporting cells obliter-

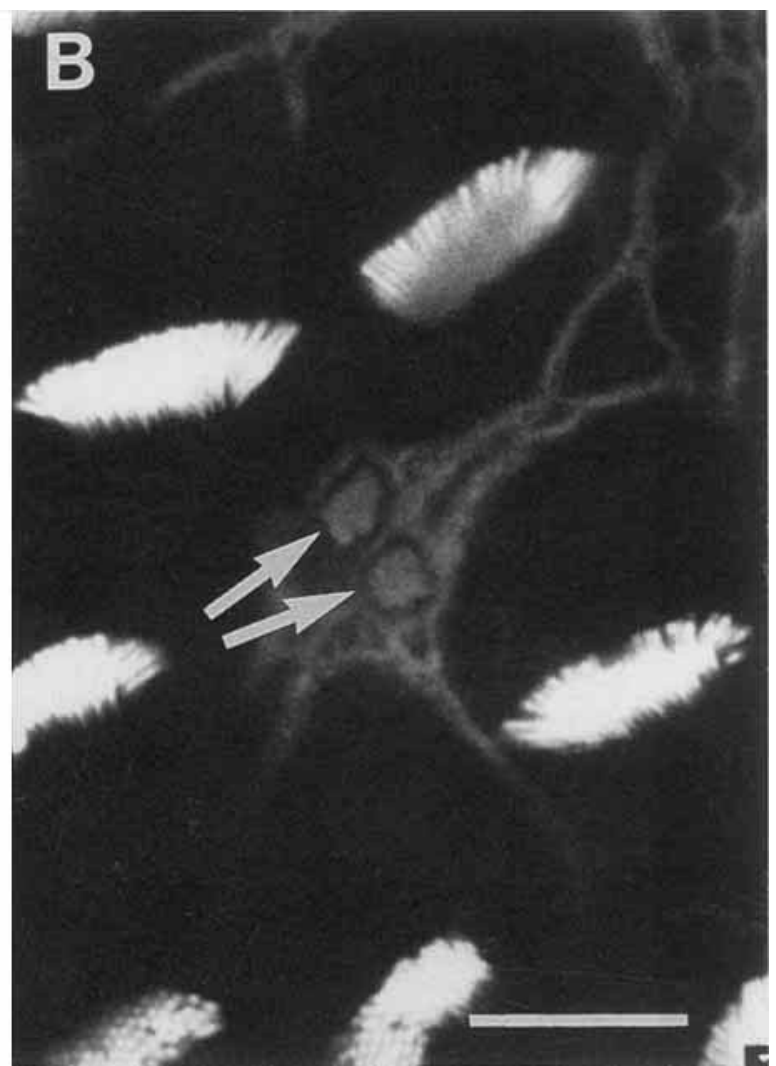

and others appear individually (short arrow). B: Laser confocal micrograph of the distal basilar papilla. A pair of small hair cells (arrows) is present among normal looking hair cells in an area where no hair cell loss is detected. Bars: $5 \mu \mathrm{m}$.

ate damaged hair cells and that the basolateral portion of degenerating hair cells remains within the epithelium (Raphael and Altschuler, '91). In contrast, dying hair cells are ejected (or extruded) from the epithelium after severe acoustic overstimulation in the chick BP (Cotanche et al., '87) and after laser microbeam irradiation in the lateral line organ (Balak et al., '90). Irradiation may affect supporting cell nuclei and abolish their ability to quickly expand and replace hair cells. Nevertheless, in view of the present results it appears that elimination of hair cells from the epithelium can occur via two different routes: 1 ) extrusion and ejection to the luminal fluid or 2) obliteration and degeneration of hair cells within the epithelial layer.

Future experiments will determine the causal relationship between the extent of trauma and the route of hair cell degeneration. I speculate that after severe trauma caused by lengthy noise exposure, hair cells may be extruded, whereas with moderate noise exposure hair cells degenerate within the epithelium and are not ejected. In either case, supporting cells appear to expand in a synchronized way, preventing discontinuities in the $\mathrm{RL}$.

\section{Reorganization of apical junctions}

A change in the apical contour of supporting cells in the distal BP was invariably observed 4 hours after the onset of noise exposure. In control tissues, the apical contour of 

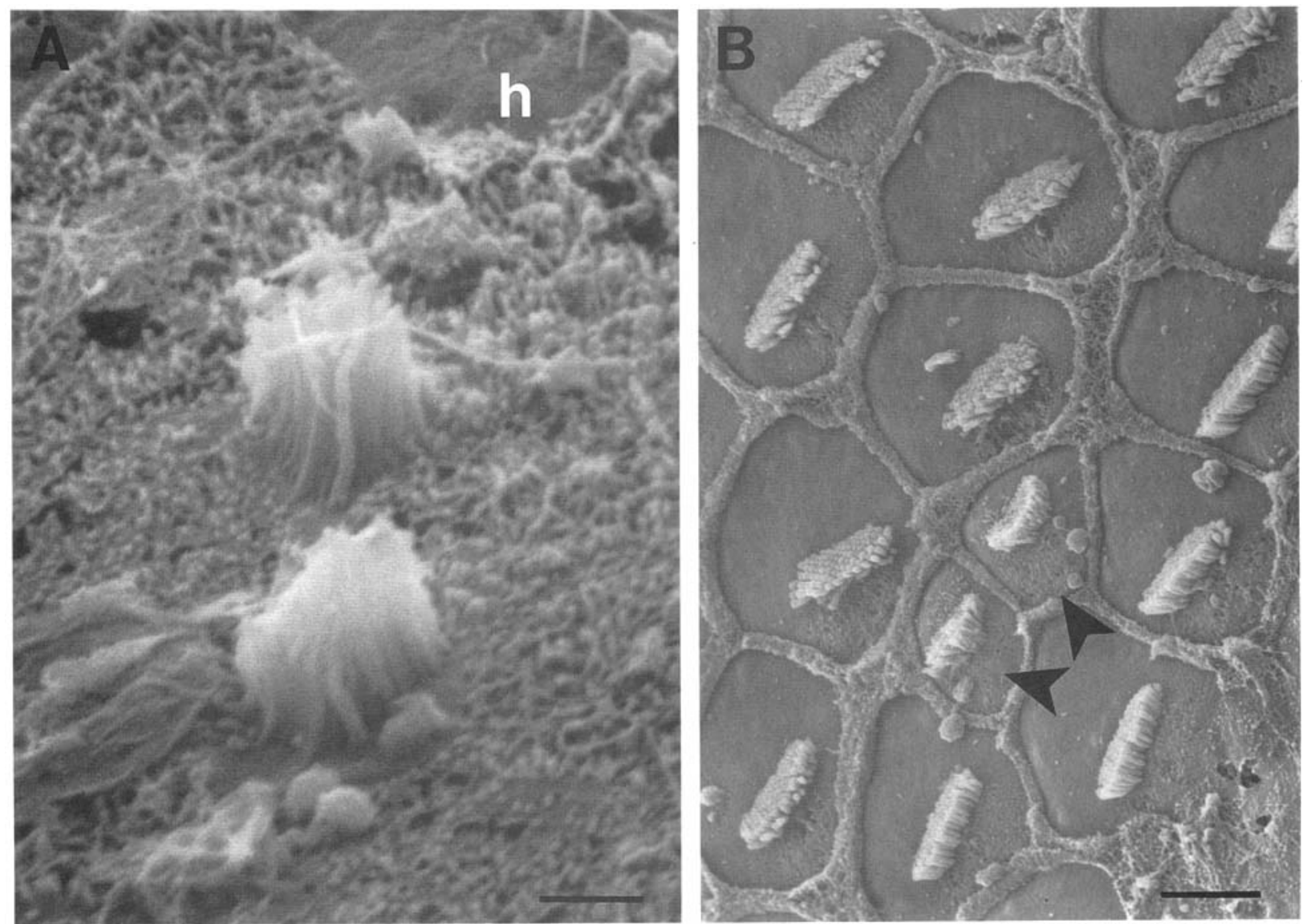

Fig. 8. SEM micrograph of the center of lesion (A) and the distal basilar papilla (B) 96 hours after noise exposure. A: A pair of small, immature hair cells is located in an area with partial hair cell loss. Large hair cells that survived the noise exposure are also seen $(h)$. The immature hair cells are oriented with the tallest stereocilia and the kinocilium facing the abneural side of the basilar papilla. B: A pair of

supporting cells appeared slender, forming a polygon around the hair cells. In contrast, supporting cells after trauma appeared as a figure eight. 'The figure eight pattern was evident with actin-specific or cingulin-specific labeling, as well as with SEM. It is unlikely that new cells played a role in altering the organization of the RL and forming the figure eight contours, since figure eights could be detected as early as 4 hours after the onset of noise exposure, a time period too short for mitosis to occur.

Double-label experiments co-localized actin with cingulin in the figure eight, indicating that elements of adherens junctions (actin) and tight junctions (cingulin) participated in creating this figure eight. It is likely, therefore, that change in the apical contour of supporting cells is accompanied by reorganization of the apical junctional complexes. I speculate that the reorganization of actin bundles in supporting cells represents a stress response of these cells, caused directly or indirectly by overstimulation. The purpose of this stress response could be to maintain the surface tension of the epithelium at the RL, or to regulate supporting cell expansion and hair cell constriction. Based on this tentative role, I propose to name the shape transition of supporting cells "SAFE" (Stress-Associated Figure Eight). immature hair cells (arrowheads) is located among mature hair cells. Preexisting hair cells are regularly arranged and no missing cells can be detected. New hair cells are oriented in register with other cells in area, with taller rows of stereocilia facing abneural side (lower left side of micrograph). Bars: $5 \mu \mathrm{m}$.

Constriction of the apical surface of hair cells is likely to result from active constriction of the circumferential actin belt associated with the adherens junction. Constricted actin belts have also been observed in outer hair cells in the organ of Corti (Raphael and Altschuler, '92). I speculate that the force and speed of hair cell constriction after noise may be directly related to the amount of mechanical stress, which in turn is a function of the severity of the trauma. Supporting cells attempt to restrain hair cell constriction, but if the contractile force of the hair cell exceeds a critical level, supporting cells expand and invade the space of the constricting hair cell. In the distal BP, overstimulation is less severe, contractile forces are weaker, and therefore supporting cells are able to restrain hair cells and prevent their contraction. The role of the SAFE in this proposed model would be to reinforce the apical domain of supporting cells, to help restrain hair cell constriction and thereby prevent hair cell loss.

\section{Is hair cell death active?}

Active cell death, also known as apoptosis, occurs as a normal type of cell death in developing and mature tissues, and may also result from trauma (Fawthrop et al., '91; 
Gerschenson and Rotello, '92; Raff, '92). It is likely that constriction of the apical actin belt in hair cells is an active process, similar to constriction of the contractile ring that separates two daughter cells from each other during cytokinesis (Schroeder, '73). If true, the apical constriction in damaged hair cells may constitute the first step in a cascade of cellular activities leading to cell death. Thus, I raise the possibility that hair cells are actively involved in their own demise. A future goal will be to determine if degenerating hair cells express any number of genes that encode apoptosisassociated gene products.

\section{Signals for supporting cell division and hair cell production}

A major finding in the present work is that new hair cells may appear in distal areas of the BP, where hair cell damage is minimal and hair cell loss could not be detected. This finding raises the possibility that hair cell loss is not necessary for production of new hair cells. It should be noted that in this work, the lack of hair cell loss has been determined by a subjective assessment based on the presence of orderly, uninterrupted rows of normal looking hair cells. This subjective assessment cannot conclusively rule out that single cells are eliminated unnoticed. Nevertheless, it is rather unlikely that two or more neighboring hair cells would be missing without being detected. Due to the cellular organization at the RL, to completely deprive a supporting cell of heterophilic contacts with hair cells, at least two hair cells must be lost (see Corwin et al., '91, for review). Thus, the data indicate that removal of lateral inhibition is not necessary for producing new hair cells in the traumatized BP. These findings corroborate previous evidence that supporting cells that divide often maintain contacts with preexisting hair cells (Raphael, '92).

Ionic leaks due to holes in the RL are also thought to constitute a signal for regeneration after hair cell loss in the BP (Corwin et al., '91). However, in this work the RL and the network of tight junctions were structurally uninterrupted during hair cell degeneration and regeneration, suggesting that changes in ionic composition do not play a role in the signalling mechanism of regeneration. Nevertheless, a direct measurement of the functional integrity of the $\mathrm{RL}$ as an ionic barrier should be performed before endolymphatic leak is unequivocally ruled out as a signal for regeneration.

In conclusion, the present work demonstrated that after intense noise exposure, damaged hair cells often display constricted apical surface area and altered apical contour. Dying hair cells may disintegrate within the epithelium while the RL remains structurally undisturbed. Structural changes in response to noise occur in supporting cells in the distal BP, where hair cell damage is minimal and hair cell loss is undetected. New hair cells are observed in the BP 4 days after acoustic trauma, in regions with or without noticeable hair cell loss. Regenerated hair cells are appropriately oriented in the BP as soon as they can be detected with the present methods.

These findings emphasize the important role of supporting cells in the course of inner ear trauma. Based on the present results, it is proposed that degenerating hair cells may undergo active cell death. It is further speculated that hair cell degeneration, supporting cell expansion, and hair cell regeneration are mediated by tensile forces at the RL and changes in cell shape. Experiments are now underway to examine these hypotheses, and to determine whether production of new hair cells in the center of the lesion is regulated by the same mechanisms as in distal regions of the BP.

\section{ACKNOWLEDGMENTS}

I offer many thanks to Peter Finger, Michael Lee, and Dr. $\mathrm{Yu}$ Wang, who provided excellent technical support. I am extremely grateful to Drs. Stephen Easter, Donna Martin, Pamela Raymond, and Kathryn Tosney for their very helpful comments on the manuseript. I thank Dr. Sandra Citi (Cornell University Medical School, New York) for kindly donating anti-cingulin antibodies. This work was supported by a grant from the Deafness Research Foundation.

\section{LITERATURE CITED}

Balak, K.J., J.T. Corwin, and J.E. Jones (1990) Regenerated hair cells can originate from supporting cell progeny: Evidence from phototoxicity and laser ablation experiments in the lateral line system. J. Neurosci. 10:2502-2512.

Calof, A.L., A.D. Landner, and D.M. Chikaraishi (1991) Regulation of neurogenesis and neuronal differentiation in primary and immortalized cells from mouse olfactory epithelium. In Regeneration of Vertebrate Sensory Receptor Cells. Ciba Foundation Symposium 160. New York: John Wiley \& Sons, p. 249-276.

Chandler, J.P. (1984) Light and electron microscopic studies of the basilar papilla in the duck, Anas platyrhynchos. I. The hatchling. J. Comp. Neurol. 222:506-522.

Citi, S., H. Sabanay, R. Jakes, B. Geiger, and J. Kendrick-Jones (1988) Cingulin, a new peripheral component of tight junctions. Nature 333:272276.

Citi, S., H. Sabanay, J. Kendrick-Jones, and B. Geiger (1989) Cingulin: Characterization and localization. J. Cell Sci. 93:107-122.

Corwin, J.'T., and D.A. Cotanche (1988) Regeneration of sensory hair cells after acoustic trauma. Science 240:1772-1774.

Corwin, J.T., J.E. Jones, A. Katayama, M.W. Kelley, and M.E. Warchol (1991) Hair cell regeneration: The identities of progenitor cells, potential triggers and instructive cues. In Regeneration of Vertebrate Sensory Receptor Cells. Ciba Foundation Symposium 160. New York: John Wiley \& Sons, p. 103-130.

Cotanche, D.A. (1987) Regeneration of hair cell stereociliary bundles in the chick cochlea following severe acoustic trauma. Hear. Res. 30:181-196.

Cotanche, D.A., and C.E.e. Dopyera (1990) Hair cell and supporting cell response to acoustic trauma in the chick cochlea. Hear. Res. 46:29-40.

Cotanche, D.A., J.C. Saunders, and L.G. Tilney (1987) Hair cell damage produced by acoustic trauma in the chick cochlea. Hear. Res. 25:267286

Fawthrop, D.J., A.R. Boobis, and D.S. Davies (1991) Mechanisms of cell death. Arch. Toxicol. 65:437-444.

Gerschenson, L.E., and R.J. Rotello (1992) Apoptosis: A different type of cell death. FASEB J. 6:2450-2455.

Girod, D.A., L.G. Duckert, and E.W Rubel (1989) Possible precursors of regenerated hair cells in the avian cochlea following acoustic trauma. Hear. Res. 42:175-194.

Graziadei, P.P., and G.A. Monti Graziadei (1985) Neurogenesis and plasticity of the olfactory sensory neurons. Ann. N.Y. Acad. Sci. 457:127-142.

Hirokawa, N. (1978) The ultrastructure of the basilar papilla of the chick. J. Comp. Neurol. 181:361-374.

Marsh, R.R., L. Xu, J.P. Moy, and J.C. Saunders (1990) Recovery of the basilar papilla following intense sound exposure in the chick. Hear. Res. $46: 229-238$.

Presson, J.C., and A.N. Popper (1990) Possible precursors to new hair cells, support cells, and Schwann cells in the ear of a post-embryonic fish. Hear. Res. 46:9-22.

Raff, M.C. (1992) Social controls on cell survival and cell death. Nature $356: 397-400$

Raphael, Y. (1991) Damage to the tectorial membrane may protect chick hair cells from noise overstimulation. Hear. Res. 53:173-184 
Raphael, Y. (1992) Evidence for supporting cell mitosis in response to acoustic trauma in the avian inner ear. J. Neurocytol. 21:663-671.

Raphael, Y., and R.A. Altschuler (1991) Modulation of cytoskeletal and junctional proteins during hair cell degeneration. Cell Motil. 18:215-227.

Raphael, Y., and R.A. Altschuler (1992) Early microflament reorganization in injured auditory epithelia. Exp. Neurol. 115:32-36.

Raymond, P.A. (1991) Retinal regeneration in teleost fish. In Regeneration of Vertebrate Sensory Receptor Cells. Ciba Foundation Symposium 160 New York: John Wiley \& Sons, p. 171-191.
Reh, T.H., M. Jones, and C. Pittack (1991) Common mechanisms of retinal regeneration in the larval frog and embryonic chick. In Regeneration of Vertebrate Sensory Receptor Cells. Ciba Foundation Symposium 160. New York: John Wiley \& Sons, p. 192-207.

Ryals, B.M., and E.W Rubel (1988) Hair cell regeneration after acoustic trauma in adult Coturnix quail. Science 240:1774-1776.

Schroeder, T.E. (1973) Actin in dividing cells: Contractile ring filaments hind heavy meromyosin. Proc. Natl. Acad. Sci. USA 70:1688-1692.

Tanaka, K., and C.A. Smith (1978) Structure of the chicken's inner ear: SEM and 'TEM study. Am. J. Anat. 153:251-271. 